\title{
Zero phase error filtering method based on diffusion equation
}

\author{
Lianyou Lai ${ }^{\mathrm{a}}$, Weijian Xu ${ }^{\mathrm{b}}$ \\ College of Information Engineering, Jimei University, Xiamen, China \\ akaikaixinxinlly@163.com, bxucomma@163.com
}

\begin{abstract}
The phase error between digital filtering results and the original signal exists. In order to solve this problem, a ZPEF (Zero Phase Error Filtering) method based on diffusion filtering equation is proposed. And the method is proposed which the absolute value of maximum gradient value is used as the stopping condition for diffusion filtering. Firstly, one dimensional signal with noise in time domain is transformed into one dimension signal in spatial domain. Then, the spatial domain signal is filtered by digital iterative method based on diffusion equation. Finally, the stopping condition of filtering process is judged according to the absolute value of the maximum gradient. For the iterative process of the algorithm, the stability condition of the algorithm is given. Experimental results show that DF method can realize the zero-phase error filter. This method is superior to the FRR (Forward-Reverse Filtering, Reverse Output) and RFF (Reverse-Reverse Filtering, Forward Output) zero phase error filter, especially in the case of small sample data.
\end{abstract}

Key words : Zero phase error filtering; Zero phase error; Diffusion filtering; Diffusion equation

\section{Introduction}

The filter also changes the phase of the signal while changing the amplitude of the signal. There is a phase difference between the filtered signal and the original signal [1-2]. The phase of the signal contains important information, even the measured object itself is phase or phase difference in many measurement situations, such as lightning impulse signal processing, zero phase error motion control, ECG signal processing, laser ranging based on phase modulation and so on. At this time, the phase difference between filtered signal and original signal is determined [3-7]. If the phase difference between the filtered signal and the original signal is zero, it indicates that the phase information of the filtered signal is well preserved and has not changed. This is the ideal case [8-9]. The best way to determine the phase difference between filtered signal and original signal is to use zero phase difference filtering method. That is, the filtered signal does not generate phase shift, and is kept in phase with the original signal before filtering.

At present, zero-phase error filtering methods have FRR and RRF that the detailed exposition is seen in the literature [10]. The principle of the FRR filtering method is: First, the original signal is filtered in sequence. Second, the order of the obtained filtered results is reversed. Then the reversed signal is filtered again. Finally, the filtered data is inverted in sequence and output. The principle of the RRF filtering method is: First, the order of the original signal is reversed. Secondly, the data filtered after the reversal is filtered. Then the order of the filtered results is reversed. Finally, the inverted signal is filtered and output again. The characteristics of FRR and RRF filtering methods are that the sequence of signals has to be reversed by 2 times, and the signal needs 2 times to pass the filter. The shortcoming of filtering FRR and REF is that there are large distortion at both ends of the filtered signal in the case of small samples. In applications, zero-phase error filtering method has been applied to non-stationary signal processing by Chang Guang.

A new zero-phase error filtering method is proposed after analysis of the filtering characteristics of the diffusion equation. Finally, the effectiveness of the method is verified by simulation experiments.

\section{ZPEF method using diffusion equation}

\section{A. The principle of $D F$}

The idea of diffusion filtering is based on multi-scale description of signals. Theoretically, it can be regarded as isotropic heat conduction or thermal diffusion process. The multi-scale filtering of the original signal $I_{0}(x, y)$ can be 
expressed as $I(x, y, t)=I_{0}(x, y) \cdot G(x, y, t)$ that is the convolution operation of the Gauss kernel $G$ with variance of time $t$ and the original signal $I_{0}(x, y)$ [12-15]. It is namely isotropic diffusion filtering.

On the basis of isotropic filtering, Perona and Malik further proposed anisotropic diffusion filtering method in 1990, that is, PM model:

$$
\left\{\begin{array}{l}
\frac{\partial I}{\partial t}=\operatorname{div}[c(\|\nabla I\|) \cdot \nabla I] \\
I(t=0)=I_{0}
\end{array}\right.
$$

Where, div is divergence operator; $\nabla$ is gradient operator; \|\| is amplitude; $c(\|\nabla I\|)$ is diffusion equation; $t$ is time operator which indicates that filtering process is related to diffusion time.

The formula (1) shows that the diffusion coefficient is a function of the gradient module. According to the relationship between gradient value and diffusion coefficient, Perona and Malik put forward two kinds of diffusion equations.

$$
\begin{gathered}
c(\|\nabla I\|)=\frac{1}{1+(\|\nabla I\| / k)^{2}} \\
c(\|\nabla I\|)=\exp \left[-(\|\nabla I\| / k)^{2}\right]
\end{gathered}
$$

Where, $\|\nabla I\|$ is the edge detector. If $\|\nabla I\| \square k$, then $c(\|\nabla I\|) \rightarrow 0$, the diffusion is suppressed. If $\|\nabla I\| \square k$, then $c(\|\nabla I\|) \rightarrow 1$, the diffusion is strengthened. $k$ can be seen as a diffusion threshold. The larger the $k$ value is, the stronger the diffusion effect is, and the signal diffused is smoother.

When $c(\|\nabla I\|)$ is a constant $k$, the equation (1) is an isotropic diffusion equation, which can be written in the form of (4).

$$
\begin{cases}\frac{\partial u}{\partial t}=k \frac{\partial^{2} u}{\partial x^{2}} & -\infty<x<\infty, t>0 \\ u(x, 0)=u_{0}(x) & -\infty<x<\infty, t=0\end{cases}
$$

In (4), $u$ is one-dimensional signal, $k$ is diffusion coefficient, and satisfies $k>0$. Its initial conditions are shown in formula (5). Its boundary conditions are shown in formula (6)

$$
u(x, 0)=u_{0}(x), 0 \leq x \leq x_{f}
$$

$$
u(0, t)=b_{0}(t), u\left(x_{f}, t\right)=b_{x f}(t), 0 \leq t \leq T
$$

In (4), $t$ is regarded as a parameter. The (4) is used as the Fourier transform of $x$. Set $F[u(x, t)]=U(\omega, t)$, $F\left[u_{0}(x)\right]=U_{0}(\omega)$, and (4) become (7)

$$
\left\{\begin{array}{l}
\frac{\mathrm{d} U(\omega, t)}{\mathrm{d} t}=-k \omega^{2} U(\omega, t) \\
U(\omega, 0)=U_{0}(\omega)
\end{array}\right.
$$

Where, (7) is a first order ordinary differential equation with parameter $\omega$. Its solution is shown in (8).

$$
U(\omega, t)=U_{0}(\omega) e^{-k \omega^{2} t}
$$

(8) is used as inverse Fourier transform, and the (9) is available.

$$
u(x, t)=F^{-1}\left[U_{0}(\omega)\right] * F^{-1}\left[e^{-k \omega^{2} t}\right]
$$

Where,

$$
\begin{gathered}
F^{-1}\left[U_{0}(\omega)\right]=u_{0}(x) \\
F^{-1}\left[e^{-k \omega^{2} t}\right]=\frac{1}{2 \sqrt{\pi k t}} e^{-\frac{x^{2}}{4 k t}}
\end{gathered}
$$

Therefore, $u(x, t)$ can be written in (12).

$$
u(x, t)=u_{0}(x) * \frac{1}{2 \sqrt{\pi k t}} e^{-\frac{x^{2}}{4 k t}}
$$

Type (12) right can be written (13).

$$
\begin{aligned}
u(x, t) & =u_{0}(x) * G_{\sigma}(x, t) \\
& =G_{\sigma}(x, t) * u_{0}(x)
\end{aligned}
$$

Where,

$$
G_{\sigma}(x, t)=\frac{1}{2 \sqrt{\pi k t}} e^{-\frac{x^{2}}{4 k t}}
$$

The (14) is written as a standard normal distribution form, as shown in (15).

$$
G_{\sigma}(x, t)=\frac{1}{\sqrt{2 \pi} \sigma} e^{-\frac{x^{2}}{2 \sigma^{2}}}
$$

Where,

$$
\begin{aligned}
4 k t & =2 \sigma^{2} \\
\sigma & =\sqrt{2 k t}
\end{aligned}
$$

The (13) shows that the result $u(x, t)$ of isotropic diffusion filtering is equal to the convolution of the initial 
noise signal $u_{0}(x)$ and the Gauss function $G_{\sigma}(x, t)$.

\section{B. Stopping condition for $D F$}

$\mathrm{DF}$ is a process of smoothing the signal. If the diffusion time is too short, the noise in the signal can not be effectively filtered. If the diffusion time is too long, the useful information of the signal will not be filtered out. In (17), If $t$ is too small, then $\sigma$ is very small. The Gauss kernel $G_{\sigma}(x, t)$ is approximated as a selective pulse. The $u(x, t)$ is approximately equal to the initial signal. If $t$ is too big, $\sigma$ is big. The Gauss kernel $G_{\sigma}(x, t)$ is approximately a straight line. $u(x, t)$ will also be smoothed into a straight line. Therefore, the stopping condition of DF is necessary. When the stopping condition meets, the diffusion process stops automatically.

In this paper, it is proposed that the absolute value of the maximum gradient is used as the judging condition for whether the DF process is stopped, as shown in (18).

$$
\max (|\operatorname{grad}(u(x, t))|)<G_{\max }
$$

In the process of DF, iterative computation is carried out over time. The signal gradually tends to be smooth. the gradient values of all calculated points gradually decrease In general. After each diffusion is completed, the absolute value of the gradient of each point is calculated. When the absolute value of the gradient is less than $G_{\max }$, the diffusion is stopped and the filtering is completed.

\section{Simulation experiment and analysis}

\section{A. Simulation of $D F$ process}

The initial signal is a sinusoidal signal with noise, and its expression is $y(t)=\sin (t)+0.5 \sin (3 t)+($ rand -0.5$)$.

Where, $($ rand -0.5$)$ is a noise with a range of 0.5 averages of 0 . The time domain signal $y(t)$ is regarded as the spatial signal $y(x)$. When the $y(x)$ is discrete, take $\Delta x=0.1, x \in[0,5 \pi]$. The $y(x)$ is simulated wth the time step $\Delta t=0.001$. The diffusion process is shown in Fig. 1.

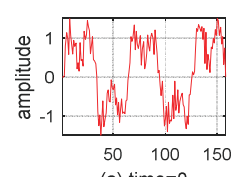

(a) time $=0$

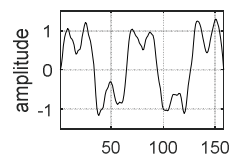

(c) time $=10 \Delta t$

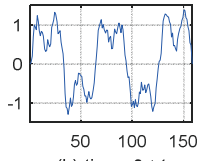

(b) time $=3 \Delta t$

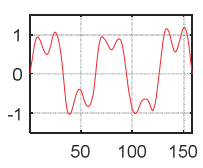

(d) time $=30 \Delta t$
Fig. 1 The process of DF

Fig. 1 shows that: (1) With the development of diffusion, the high frequency noise is smoothed gradually, and the function of filtering is realized. (2) Before and after diffusion filtering, the phase of the signal has not changed.

\section{B. Simulation results and analysis}

The ZPEF method proposed in this paper is simulated and compared with the traditional FRR and RRF zero phase error filtering methods.

Example 1: Simulation of general noisy signal

The initial signal is a sinusoidal signal with noise. The expression is $y(t)=\sin (t)+0.5 \sin (3 t)+1.5($ rand -0.5$)$.

Where, (rand-0.5) is a noise with a range of 0.5 and averages of 0 . Takes $t \in[0,3 \pi], N=100$. The stopping condition of DF is $G_{\max }=0.22$. The result simulated is shown in Fig. 2.

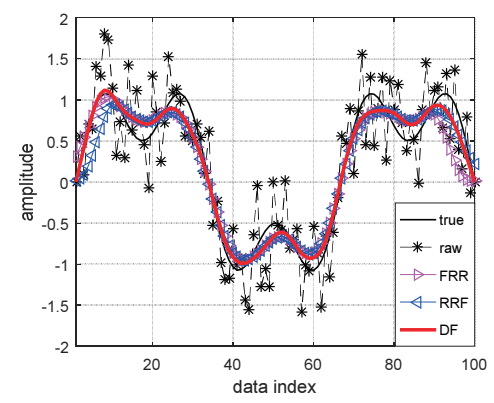

Fig. 2 Diffusion filter

Fig. 2 shows that: (1) DF effectively filters out the noise in the data, and the filtering effect is very good. (2) The phase of the signal before and after DF has not been changed. It means that ZPEF has been realized. (3) The effect of DF is better than that of FRR and RRF. At the beginning and end of the data, DF is better than FRR and 
RRF in maintaining the true shape of the signal. The distortion of FRR and RRF filtering results is here. The result of the FRR and RRF are both distorted.

Example 2: Simulation in small sample case

The initial signal is a sinusoidal signal with noise. The expression is $y(t)=1.5 \sin (t)+1.5($ rand -0.5$)$.

(rand-0.5) is a noise with a range of 0.5 and averages of

0 . Takes $t \in[0,3 \pi], N=30$. The stopping condition of $\mathrm{DF}$ is $G_{\max }=0.22$. The result of the simulation is shown in Fig. 3.

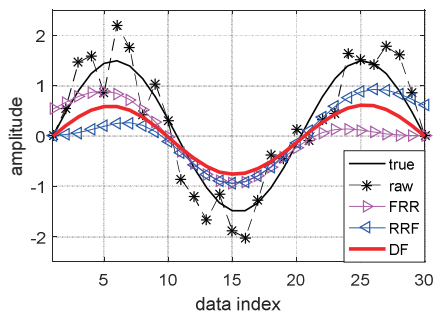

Fig.3 Diffusion filter (initial signal: the small sample sine wave with noise)

As you can see in Fig. 3, the filtered data of DF better maintain the true form of the signal at the head and tail of the filtered signals.But the results of the FRR and RRF are distorted. In the case of small samples, this advantage is more significant.

\section{Conclusion}

The process of DF is: (1) First, a time domain data is collected, (2) Secondly, it is regarded as an airspace data, (3) Then, the raw data is filtered by the diffusion equation. DF can achieve zero phase error filtering. DF is better compared with FRR and RRF filtering. At the beginning and end of the data, the filtered data by DF is slightly distorted than those by the FRR and RRF. This advantage is even more evident when ZPEF is used for small sample data.

\section{References}

[1] YAN S, SUN L and XU L. "2-D zero-phase IIR notch filters design based on state-space representation of 2-D frequency transformation". IEEE International Symposium on Circuits and Systems, IEEE, vol. 42, pp. 2369-2370, 2015.

[2] YANG D Y, WANG B and CAI G W. "Study on the harmonic detection method based on improved adaptive local iterative filtering". Chinese Journal of Scientific Instrument, vol. 54, pp. 2274-2281, 2016.

[3] ZHANG H M and HAN W G. "A new variable step LMS algorithm and its application". Chinese Journal of Scientific Instrument, vol. 41, pp. 1822-1830, 2015.

[4] XIE Y H and LI L. "Covariance object tracking algorithm based on bilateral filtering". Chinese Journal of Measurement and Instrument, vol. 46, pp. 1282-1287, 2016.

[5] LI Q, ZHU L and SHAO W Q. "Anisotropic diffusion filtering algorithm for SAR images". Foreign Electronic Measurement Technology, vol. 42, pp. 59-64, 2016.

[6] JI Yuebo and QIN Shuren. "Digital filtering with zero phase error". Journal of Chongqing University: Natural Science Edition, vol. 26, pp. 4-6, 2010.

[7] CHANG Guang and YAN Suyun. "Application of zero-phase digital filter on non-stationary signal processing". Journal of Beijing Jiaotong University, vol. 35, pp. 49-56, 2011.

[8] FENG L B, ZHUANG P and LIU F. "Finite element method for space-time fractional diffusion equation". Numerical Algorithms, vol. 72, pp. 749-767, 2016.

[9] TIAN W, MA T and ZHENG Y. "Weighted curvature-preserving PDE image filtering method". Computers \& Mathematics with Applications, vol. 70, pp. 1336-1344, 2015.

[10] HODNELAND E, TAI X and KALISCH H. "PDE based algorithms for smooth watersheds". IEEE Transactions on Medical Imaging, vol. 35, pp. 957-966, 2016. 